\title{
Stokes operators with variable coefficients and applications
}

Veli B Shakhmurov*

\author{
"Correspondence: \\ veli.sahmurov@okan.edu.tr \\ Department of Mechanical \\ Engineering, Okan University, \\ Akfirat, Tuzla, Istanbul 34959, Turkey \\ Institute of Mathematics and \\ Mechanics, Azerbaijan National \\ Academy of Sciences, Baku, \\ Azerbaijan
}

\begin{abstract}
The stationary and instationary Stokes problems with variable coefficients in abstract $L^{p}$ spaces are considered. The problems contain abstract operators and nonlocal boundary conditions. The well-posedness of these problems is derived.

MSC: 35Q30; 76D05; 34G10; 35J25

Keywords: Stokes systems; Navier-Stokes equations; abstract differential equations with variable coefficients; semigroups of operators; boundary value problems; maximal $L^{p}$ regularity
\end{abstract}

\section{Introduction}

We consider the initial and boundary value problem (BVP) for the following Stokes type equation with variable coefficients:

$$
\begin{aligned}
& \frac{\partial u}{\partial t}+\sum_{k=1}^{n} a_{k}(x) \frac{\partial^{2} u}{\partial x_{k}^{2}}+A(x) u+\sum_{k=1}^{n} A_{k}(x) \frac{\partial u}{\partial x_{k}}+\nabla \varphi=f(x, t), \quad \operatorname{div} u=0, \\
& \sum_{i=0}^{m_{k j}}\left[\alpha_{k j i} \frac{\partial^{i} u}{\partial x_{k}^{i}}\left(G_{k 0}\right)+\beta_{k j i} \frac{\partial^{i} u}{\partial x_{k}^{i}}\left(G_{k b}\right)\right]=0, \quad k=1,2, \ldots, n, j=1,2, \\
& u(x, 0)=a(x), \quad x \in G, t \in(0, T),
\end{aligned}
$$

where

$$
\begin{aligned}
& x=\left(x_{1}, x_{2}, \ldots, x_{n}\right) \in G=\prod_{k=1}^{n}\left(0, b_{k}\right), \quad m_{k j} \in\{0,1\}, \\
& G_{k 0}=\left(x_{1}, x_{2}, \ldots, x_{k-1}, 0, x_{k+1}, \ldots, x_{n}\right) \\
& G_{k b}=\left(x_{1}, x_{2}, \ldots, x_{k-1}, b_{k}, x_{k+1}, \ldots, x_{n}\right)
\end{aligned}
$$

$A=A(x)$ and $A_{k}=A_{k}(x)$ are linear operators in a Banach space $E, \alpha_{k j i}, \beta_{k j i}$ are complex numbers, and $a_{k}$ are complex-valued functions. Here $f=\left(f_{1}(x, t), f_{2}(x, t), \ldots, f_{n}(x, t)\right)$ represents a given, $a$ denotes the initial data and

$$
u=\left(u_{1}(x, t), u_{2}(x, t), \ldots, u_{n}(x, t)\right), \quad \varphi=\varphi(x, t)
$$

(02014 Shakhmurov; licensee Springer. This is an Open Access article distributed under the terms of the Creative Commons Attribution License (http://creativecommons.org/licenses/by/2.0), which permits unrestricted use, distribution, and reproduction in any medium, provided the original work is properly cited. 
represent the unknown functions. Moreover, $u_{j}(x, t), f_{j}(x, t)$, and $\varphi(x, t)$ are $E$-valued functions. This problem is characterized by the presence of abstract operator functions and complex-valued variable coefficients in the principal part. Moreover, boundary conditions are nonlocal, generally. The existence, uniqueness, and coercive estimates of maximal regular solution of problem (1.1)-(1.2) are obtained. Since the Banach space $E$ is arbitrary and $A$ is a possible linear operator, by choosing $E$ and operators $A, A_{k}$ we can obtain maximal regularity properties for numerous class of Stokes type problems with variable coefficients. For $E=\mathbb{C}, a_{k}(x) \equiv-1, A=\varkappa>0$ problem (1.1)-(1.2) is reduced to nonlocal Stokes problem

$$
\begin{aligned}
& \frac{\partial u}{\partial t}-\Delta u+\varkappa u+\nabla \varphi=f(x, t), \quad \operatorname{div} u=0, \\
& \sum_{i=0}^{m_{k j}}\left[\alpha_{k j i} \frac{\partial^{i} u}{\partial x_{k}^{i}}\left(G_{k 0}, t\right)+\beta_{k j i} \frac{\partial^{i} u}{\partial x_{k}^{i}}\left(G_{k b}, t\right)\right]=0, \\
& u(x, 0)=a(x), \quad x \in G, t \in(0, T), k=1,2, \ldots, n, j=1,2,
\end{aligned}
$$

where $\mathbb{C}$ is the set of complex numbers. Note that the existence of weak or strong solutions and regularity properties for the classical Stokes problems were extensively studied, e.g., in [1-10]. There is an extensive literature on the solvability of the initial value problems (IVPs) for the Stokes equation (see, e.g., $[1,2,10]$ and further papers cited there). Solonnikov [8] proved that for every $f \in L^{p}\left(\Omega \times(0, T) ; R^{3}\right)=B(p), p \in(1, \infty)$ the instationary Stokes problem

$$
\begin{aligned}
& \frac{\partial u}{\partial t}-\Delta u+\nabla \varphi=f(x, t), \quad \operatorname{div} u=0,\left.\quad u\right|_{\partial \Omega}=0, \\
& u(x, 0)=0, \quad x \in \Omega, t \in(0, T)
\end{aligned}
$$

has a unique solution $(u, \nabla \varphi)$ so that

$$
\left\|\frac{\partial u}{\partial t}\right\|_{B(p)}+\left\|\nabla^{2} u\right\|_{B(p)}+\|\nabla \varphi\|_{B(p, q)} \leq C\|f\|_{B(p, q)}
$$

Then Giga and Sohr [2] improved the result of Sollonikov for spaces with different exponents in space and time, i.e., they proved that for every $f \in L^{p}\left(0, T ;\left(L^{q}(\Omega)\right)^{n}\right)$ there is a unique solution $(u, \nabla \varphi)$ of problem (1.5) so that

$$
\left\|\frac{\partial u}{\partial t}\right\|_{B(p, q)}+\left\|\nabla^{2} u\right\|_{B(p, q)}+\|\nabla \varphi\|_{B(p, q)} \leq C\|f\|_{B(p, q)}
$$

where

$$
B(p, q)=L^{p}\left(0, T ;\left(L^{q}(\Omega)\right)^{n}\right), \quad p, q \in(1, \infty) .
$$

Moreover, the estimate obtained was global in time, i.e., the constant $C=C(\Omega, p, q)$ is independent of $T$ and $f$. To derive the global $L^{p}-L^{q}$ estimates (1.6), Giga and Sohr used abstract parabolic semigroup theory in UMD spaces. The estimate (1.6) allows one to study the existence of solution and regularity properties of the corresponding Navier-Stokes problem 
(see, e.g., [4]). Consider first at all, the stationary version of problem (1.1)-(1.3), i.e., consider the abstract Stokes problem

$$
\begin{aligned}
& \sum_{k=1}^{n} a_{k}(x) \frac{\partial^{2} u}{\partial x_{k}^{2}}+(A(x)+\lambda) u+\sum_{k=1}^{n} A_{k}(x) \frac{\partial u}{\partial x_{k}}+\nabla \varphi=f(x), \quad \operatorname{div} u=0, \quad x \in G, \\
& \sum_{i=0}^{m_{k j}}\left[\alpha_{k j i} \frac{\partial^{i} u}{\partial x_{k}^{i}}\left(G_{k 0}\right)+\beta_{k j i} \frac{\partial^{i} u}{\partial x_{k}^{i}}\left(G_{k b}\right)\right]=0, \quad k=1,2, \ldots, n, j=1,2,
\end{aligned}
$$

$\lambda$ is a complex number. By applying the corresponding projection transformation $P$, (1.7)(1.8) can be reduced to the following problem:

$$
\begin{aligned}
& P \sum_{k=1}^{n} a_{k}(x) \frac{\partial^{2} u}{\partial x_{k}^{2}}+P(A(x)+\lambda) u+P \sum_{k=1}^{n} A_{k}(x) \frac{\partial u}{\partial x_{k}}=f(x), \quad x \in G, \\
& L_{k j} u=\sum_{i=0}^{m_{k j}}\left[\alpha_{k j i} \frac{\partial^{i} u}{\partial x_{k}^{i}}\left(G_{k 0}\right)+\beta_{k j i} \frac{\partial^{i} u}{\partial x_{k}^{i}}\left(G_{k b}\right)\right]=0, \quad k=1,2, \ldots, n, j=1,2 .
\end{aligned}
$$

Let $O_{q}$ denote the operator generated by problem (1.9)-(1.10), i.e., let $O_{q}$ be a Stokes operator in the $E$-valued solenoidal space $L_{\sigma}^{q}(G ; E)$ defined by

$$
\begin{aligned}
& D\left(O_{q}\right)=\left(W_{\sigma}^{2, q}(G ; E(A), E)\right)^{n}=\left\{u \in\left(W^{2, q}(G ; E(A), E)\right)^{n}, L_{k j} u=0, \operatorname{div} u=0\right\}, \\
& O_{q} u=P \sum_{k=1}^{n} a_{k}(x) \frac{\partial^{2} u}{\partial x^{2}} u+P A u+P \sum_{k=1}^{n} A_{k}(x) \frac{\partial u}{\partial x_{k}} .
\end{aligned}
$$

We prove that $O_{q}$ is a positive operator and $-O_{q}$ is a generator of an analytic semigroup in $L_{\sigma}^{q}(G ; E)$. In other words, we consider the instationary Stokes problem (1.1)-(1.3) and prove the well-posedness of this problem. We prove that there is a unique solution $(u, \nabla \varphi)$ of problem (1.1)-(1.3) for $f \in\left(L^{p}\left(0, T ; X_{q}\right)\right)^{n}, a \in Y_{p, q}$, and the following estimate holds:

$$
\left\|\frac{\partial u}{\partial t}\right\|_{L^{p, q}}+\sum_{k=1}^{n}\left\|\frac{\partial^{2} u}{\partial x_{k}^{2}}\right\|_{L^{p, q}}+\|A u\|_{L^{p, q}}+\|\nabla \varphi\|_{L^{p, q}} \leq C\left(\|f\|_{L^{p, q}}+\|a\|_{Y_{p, q}}\right),
$$

where $X_{q}$ is the class of $E$-valued $L^{q}$-spaces and $Y_{p, q}$ is a corresponding interpolation space. The estimate (1.11) allows one to study the existence of solution and regularity properties of the corresponding Navier-Stokes problem. Finally, we give some application of this abstract Stokes problem to anisotropic Stokes equations and systems of equations. Note that the abstract Stokes problem with constant coefficients was studied in [11].

\section{Definitions and background}

Let $E$ be a Banach space. $L^{p}(\Omega ; E)$ denotes the space of strongly measurable $E$-valued functions that are defined on the measurable subset $\Omega \subset R^{n}$ with the norm

$$
\|f\|_{L^{p}}=\|f\|_{L^{p}(\Omega ; E)}=\left(\int_{\Omega}\|f(x)\|_{E}^{p} d x\right)^{\frac{1}{p}}, \quad 1 \leq p<\infty
$$


The Banach space $E$ is called an UMD space if the Hilbert operator

$$
(H f)(x)=\lim _{\varepsilon \rightarrow 0} \int_{|x-y|>\varepsilon} \frac{f(y)}{x-y} d y
$$

is bounded in $L^{p}(R, E), p \in(1, \infty)$ (see, e.g., [12]). UMD spaces include e.g. $L^{p}, l_{p}$ spaces, and Lorentz spaces $L_{p q}, p, q \in(1, \infty)$.

Let

$$
\begin{aligned}
& S_{\psi}=\{\lambda \in \mathbb{C},|\arg \lambda| \leq \psi, 0 \leq \psi<\pi\}, \\
& S_{\psi, \varkappa}=\left\{\lambda \in S_{\psi},|\lambda|>\varkappa>0\right\} .
\end{aligned}
$$

A linear operator $A$ is said to be $\psi$-positive in a Banach space $E$ with bound $M>0$ if $D(A)$ is dense on $E$ and $\left\|(A+\lambda I)^{-1}\right\|_{B(E)} \leq M(1+|\lambda|)^{-1}$ for any $\lambda \in S_{\psi}, 0 \leq \psi<\pi$, where $I$ is the identity operator in $E$, and $B(E)$ is the space of bounded linear operators in $E$. It is known [13, $\$ 1.15 .1]$ that there exist fractional powers $A^{\theta}$ of a positive operator $A$. Let $E\left(A^{\theta}\right)$ denote the space $D\left(A^{\theta}\right)$ with norm

$$
\|u\|_{E\left(A^{\theta}\right)}=\left(\|u\|^{p}+\left\|A^{\theta} u\right\|^{p}\right)^{\frac{1}{p}}, \quad 1 \leq p<\infty, 0<\theta<\infty .
$$

Let $E_{1}$ and $E_{2}$ be two Banach spaces. By $\left(E_{1}, E_{2}\right)_{\theta, p}, 0<\theta<1,1 \leq p \leq \infty$, will be denoted the interpolation spaces obtained from $\left\{E_{1}, E_{2}\right\}$ by the $K$-method [13, \$1.3.2].

Let $\mathbb{N}$ denote the set of natural numbers. A set $\Phi \subset B\left(E_{1}, E_{2}\right)$ is called $R$-bounded (see, e.g., [14]) if there is a positive constant $C$ such that for all $T_{1}, T_{2}, \ldots, T_{m} \in \Phi$ and $u_{1}, u_{2}, \ldots, u_{m} \in E_{1}, m \in \mathbb{N}$,

$$
\int_{\Omega}\left\|\sum_{j=1}^{m} r_{j}(y) T_{j} u_{j}\right\|_{E_{2}} d y \leq C \int_{\Omega}\left\|\sum_{j=1}^{m} r_{j}(y) u_{j}\right\|_{E_{1}} d y,
$$

where $\left\{r_{j}\right\}$ is a sequence of independent symmetric $\{-1,1\}$-valued random variables on $\Omega$. The smallest $C$ for which the above estimate holds is called a $R$-bound of the collection $\Phi$ and denoted by $R(\Phi)$.

A set $\Phi_{h} \subset B\left(E_{1}, E_{2}\right)$ is called uniform $R$-bounded in $h$, if there is a constant $C$ independent on $h \in \sigma \subset \mathbb{R}$ such that

$$
\int_{\Omega}\left\|\sum_{j=1}^{m} r_{j}(y) T_{j}(h) u_{j}\right\|_{E_{2}} d y \leq C \int_{\Omega}\left\|\sum_{j=1}^{m} r_{j}(y) u_{j}\right\|_{E_{1}} d y
$$

for all $T_{1}(h), T_{2}(h), \ldots, T_{m}(h) \in \Phi_{h}$ and $u_{1}, u_{2}, \ldots, u_{m} \in E_{1}, m \in \mathbb{N}$. It is implied that $\sup _{h \in Q} R\left(\Phi_{h}\right) \leq C$.

The $\psi$-positive operator $A$ is said to be $R$-positive in a Banach space $E$ if the set $L_{A}=$ $\left\{\xi(A+\xi)^{-1}: \xi \in S_{\psi}\right\}, 0 \leq \psi<\pi$ is $R$-bounded.

The operator $A(t)$ is said to be $\psi$-positive in $E$ uniformly with respect to $t$ with bound $M>0$ if $D(A(t))$ is independent on $t, D(A(t))$ is dense in $E$ and $\left\|(A(t)+\lambda)^{-1}\right\| \leq \frac{M}{1+|\lambda|}$ for all $\lambda \in S_{\psi}, 0 \leq \psi<\pi$, where $M$ does not depend on $t$ and $\lambda$. 
Let $E_{0}$ and $E$ be two Banach spaces and $E_{0}$ continuously and densely embedded into $E$. Let $\Omega$ be a domain in $R^{n}$ and $m$ is a positive integer. $W^{m, p}\left(\Omega ; E_{0}, E\right)$ denotes the space of all functions $u \in L^{p}\left(\Omega ; E_{0}\right)$ that have generalized derivatives $\frac{\partial^{m} u}{\partial x_{k}^{m}} \in L^{p}(\Omega ; E)$ with the norm

$$
\|u\|_{W^{m, p}\left(\Omega ; E_{0}, E\right)}=\|u\|_{L^{p}\left(\Omega ; E_{0}\right)}+\sum_{k=1}^{n}\left\|\frac{\partial^{m} u}{\partial x_{k}^{m}}\right\|_{L^{p}(\Omega ; E)}<\infty .
$$

For $n=1, \Omega=(a, b), a, b \in R$ the space $W^{m, p}\left(\Omega ; E_{0}, E\right)$ will be denoted by $W^{m, p}\left(a, b ; E_{0}, E\right)$. For $E_{0}=E$ the space $W^{m, p}\left(\Omega ; E_{0}, E\right)$ is denoted by $W^{m, p}(\Omega ; E)$.

Let $L^{s, p}(\Omega ; E),-\infty<s<\infty$, denote the $E$-valued Liouville space of order $s$ such that $L^{0, p}(\Omega ; E)=L^{p}(\Omega ; E)$. It is known that if $E$ is a UMD space, then $L^{m, p}(\Omega ; E)=W^{m, p}(\Omega ; E)$ for positive integer $m$ (see, e.g., [15, \$15].

Let $L^{s, p}\left(\Omega ; E_{0}, E\right)$ denote a Liouville-Lions type space, i.e.,

$$
\begin{aligned}
L^{s, p}\left(\Omega ; E_{0}, E\right)= & \left\{u \in L^{s, p}(\Omega ; E) \cap L^{q}\left(\Omega ; E_{0}\right),\right. \\
& \left.\|u\|_{L^{s, p}\left(\Omega ; E_{0}, E\right)}=\|u\|_{L^{p}\left(\Omega ; E_{0}\right)}+\|u\|_{L^{s, p}(\Omega ; E)}<\infty\right\} .
\end{aligned}
$$

$L_{\sigma}^{q}(\Omega ; E)$ denote the $E$-valued solenoidal space, i.e., the closure of $\left(C_{0 \sigma}^{\infty}(\Omega ; E)\right)^{n}$ in $\left(L^{q}(\Omega ; E)\right)^{n}$, where

$$
C_{0 \sigma}^{\infty}(\Omega ; E)=\left\{u \in C_{0}^{\infty}(\Omega ; E), \operatorname{div} u=0\right\} .
$$

Sometimes we use one and the same symbol $C$ without distinction in order to denote positive constants which may differ from each other even in a single context. When we want to specify the dependence of such a constant on a parameter, say $\alpha$, we write $C_{\alpha}$.

The embedding theorems in vector-valued spaces play a key role in the theory of DOEs. For estimating lower order derivatives we use the following embedding theorems from [16].

Theorem $\mathbf{A}_{1}$ Suppose the following conditions are satisfied:

(1) $E$ is a UMD space and $A$ is an R-positive operator in $E$;

(2) $\alpha=\left(\alpha_{1}, \alpha_{2}, \ldots, \alpha_{n}\right)$ and $m$ is a positive integer such that $\varkappa=\sum_{k=1}^{n} \frac{|\alpha|}{m} \leq 1$, $0 \leq \mu \leq 1-\varkappa, 1<p<\infty, 0<h \leq h_{0}, h_{0}$ is a fixed positive number;

(3) $\Omega \subset R^{n}$ is a region such that there exists a bounded linear extension operator from $W^{m, p}(\Omega ; E(A), E)$ to $W^{m, p}\left(R^{n} ; E(A), E\right)$.

Then the embedding $D^{\alpha} W^{m, p}(\Omega ; E(A), E) \subset L^{p}\left(\Omega ; E\left(A^{1-\varkappa-\mu}\right)\right)$ is continuous and for all $u \in W^{m, p}(\Omega ; E(A), E)$ the following uniform estimate holds:

$$
\left\|D^{\alpha} u\right\|_{L^{p}\left(\Omega ; E\left(A^{1-\varkappa-\mu}\right)\right)} \leq h^{\mu}\|u\|_{W^{m, p}(\Omega ; E(A), E)}+h^{-(1-\mu)}\|u\|_{L^{p}(\Omega ; E)} .
$$

Remark 2.1 If $\Omega \subset R^{n}$ is a region satisfying the strong $l$-horn condition (see [4, $\left.\mathbb{\$} 7\right]$ ), $E=R$, $A=I$, then for $p \in(1, \infty)$ there exists a bounded linear extension operator from $W^{m, p}(\Omega)=$ $W^{m, p}(\Omega ; R, R)$ to $W^{m, p}\left(R^{n}\right)=W^{m, p}\left(R^{n} ; R, R\right)$. 
Theorem $\mathbf{A}_{2}$ Suppose all conditions of Theorem $\mathrm{A}_{1}$ are satisfied and $0<\mu \leq 1-\varkappa$. Moreover, let $\Omega$ be a bounded region and $A^{-1} \in \sigma_{\infty}(E)$. Then the embedding

$$
D^{\alpha} W^{m, p}(\Omega ; E(A), E) \subset L^{p}\left(\Omega ; E\left(A^{1-\varkappa-\mu}\right)\right)
$$

is compact.

Theorem $\mathbf{A}_{3}$ Suppose all conditions of Theorem $\mathrm{A}_{1}$ satisfied and $0<\mu \leq 1-\varkappa$. Then the embedding

$$
D^{\alpha} W^{m, p}(\Omega ; E(A), E) \subset L^{p}\left(\Omega ;(E(A), E)_{\varkappa, p}\right)
$$

is continuous and there exists a positive constant $C_{\mu}$ such that for all $u \in W_{p}^{l}(\Omega ; E(A), E)$ the uniform estimate holds

$$
\left\|D^{\alpha} u\right\|_{L^{p}\left(\Omega ;(E(A), E)_{\varkappa, p)}\right.} \leq C_{\mu}\left[h^{\mu}\|u\|_{W^{m, p}(\Omega ; E(A), E)}+h^{-(1-\mu)}\|u\|_{L^{p}(\Omega ; E)}\right] .
$$

From [17, Theorem 2.1] we obtain the following.

Theorem $\mathbf{A}_{4}$ Let $E$ be a Banach space, $A$ be a $\varphi$-positive operator in $E$ with bound $M$, $0 \leq \varphi<\pi$. Let $m$ be a positive integer, $1<p<\infty$ and $\alpha \in\left(\frac{1}{2 p}, \frac{1}{2 p}+m\right)$. Then, for $\lambda \in S_{\varphi}$, an operator $-A_{\lambda}^{\frac{1}{2}}$ generates a semigroup $e^{-x A_{\lambda}^{\frac{1}{2}}}$ which is holomorphic for $x>0$. Moreover, there exists a positive constant $C$ (depending only on $M, \varphi, m, \alpha$ and $p$ ) such that for every $u \in\left(E, E\left(A^{m}\right)\right)_{\frac{\alpha}{m}-\frac{1}{2 m p}, p}$ and $\lambda \in S_{\varphi}$,

$$
\int_{0}^{\infty}\left\|A_{\lambda}^{\alpha} e^{-x A_{\lambda}^{\frac{1}{2}}} u\right\|^{p} d x \leq C\left[\|u\|_{\left(E, E\left(A^{m}\right)\right)_{\frac{\alpha}{m}-\frac{1}{2 m p}, p}^{p}}^{p}+|\lambda|^{\alpha p-\frac{1}{2}}\|u\|_{E}^{p}\right] .
$$

\section{The stationary Stokes system with variable coefficients}

In this section, we derive the maximal regularity properties of the stationary abstract Stokes problem (1.7)-(1.8).

First at all, we consider the BVP for the variable coefficient differential operator equation (DOE)

$$
\begin{aligned}
& \sum_{k=1}^{n} a_{k}(x) \frac{\partial^{2} u}{\partial x_{k}^{2}}+(A(x)+\lambda) u+\sum_{k=1}^{n} A_{k}(x) \frac{\partial u}{\partial x_{k}}=f(x), \quad x \in G, \\
& \sum_{i=0}^{m_{k j}}\left[\alpha_{k j i} \frac{\partial^{i} u}{\partial x_{k}^{i}}\left(G_{k 0}\right)+\beta_{k j i} \frac{\partial^{i} u}{\partial x_{k}^{i}}\left(G_{k b}\right)\right]=0, \quad k=1,2, \ldots, n, j=1,2,
\end{aligned}
$$

where $A(x)$ and $A_{k}(x)$ are linear operators in a Banach space $E, a_{k}$ are complex-valued functions, and $\lambda$ is a complex parameter.

Maximal regularity properties for DOEs studied, e.g., in [1, 11, 12, 14, 16-24]. Nonlocal BVPs for PDE were studied in [25].

Let $\omega_{k i}=\omega_{k i}(x), i=1,2$, be roots of the equations

$$
a_{k}(x) \omega^{2}=1, \quad k=1,2, \ldots, n .
$$


Let $\alpha_{k j}=\alpha_{k j m_{k}}$ and $\beta_{k j}=\beta_{k j m_{k}}$ and

$$
\eta_{k}(x)=\left|\begin{array}{ll}
\alpha_{k 1}\left(-\omega_{k 1}\right)^{m_{k 1}} & \beta_{k 1} \omega_{k 1}^{m_{k 1}} \\
\alpha_{k 2}\left(-\omega_{k 1}\right)^{m_{k 2}} & \beta_{k 2} \omega_{k 2}^{m_{k 2}}
\end{array}\right|
$$

\section{Condition 3.1 Assume:}

(1) $E$ is a UMD space and $A(x)$ is a uniformly $R$-positive operator in $E$ for $\varphi \in[0, \pi)$;

(2) $a_{k}(x) \in C(\bar{G}), a_{k}\left(G_{i 0}\right)=a_{k}\left(G_{i b}\right), a_{k} \neq 0, a_{k} \in S\left(\varphi_{0}\right) \cap \mathbb{C} / \mathbb{R}_{-}$for all $x \in G, \varphi+\varphi_{0}<\pi$;

(3) $A(x) A^{-1}(\bar{x}) \in C(\bar{G} ; B(E)), A\left(G_{i 0}\right)=A\left(G_{i b}\right), A_{i}(x) A^{-\left(\frac{1}{2}-v\right)}(x) \in C^{(m)}(\bar{G} ; B(E)), 0<v<\frac{1}{2}$;

(4) $\left|\alpha_{k j m_{j}}\right|+\left|\beta_{k j m_{j}}\right|>0, \eta_{k}(x) \neq 0, k, i=1,2, \ldots, n, j=1,2, p \in(1, \infty)$.

Remark 3.1 Let $a_{k}=-b_{k}(x)$, where $b_{k}$ are real-valued positive functions and. Then Condition 3.1 is satisfied.

Remark 3.2 The conditions $a_{k}\left(G_{i 0}\right)=a_{k}\left(G_{i b}\right), A\left(G_{i 0}\right)=A\left(G_{i b}\right)$ are given due to the nonlocality of the boundary conditions. For local boundary conditions these assumptions are not required.

From [19, Theorem 4.1] we have the following.

Theorem 3.1 Suppose Condition 3.1 is satisfied. Then problem (3.1) has a unique solution $u \in W^{2, p}(G ; E(A), E)$ for $f \in L^{p}(G ; E)$ and for sufficiently large $\lambda \in S_{\varphi}$. Moreover, the following coercive uniform estimate holds:

$$
\sum_{k=1}^{n} \sum_{i=0}^{2}|\lambda|^{1-\frac{i}{2}}\left\|\frac{\partial^{i} u}{\partial x_{k}^{i}}\right\|_{L^{q}(G ; E)}+\|A u\|_{L^{q}(G ; E)} \leq C\|f\|_{L^{q}(G ; E)}
$$

Consider the differential operator $Q=Q_{q}$ in $L^{q}(G ; E)$ generated by problem (1.7)-(1.8), i.e.,

$$
D(Q)=W^{2, q}\left(G ; E(A), E, L_{k j}\right), \quad Q u=\sum_{k=1}^{n} a_{k}(x) \frac{\partial^{2} u}{\partial x^{2}}+A(x)+\sum_{k=1}^{n} A_{k}(x) \frac{\partial u}{\partial x_{k}} .
$$

Let $B_{q}=B\left(L^{q}(G ; E)\right)$. From Theorem 3.1 we obtain the following.

Result 3.1 For $\lambda \in S_{\psi, \varkappa}$ there is a resolvent $(Q+\lambda)^{-1}$ and the following uniform coercive estimate holds:

$$
\sum_{k=1}^{n} \sum_{i=0}^{2}|\lambda|^{1-\frac{i}{2}}\left\|\frac{\partial^{i}}{\partial x_{k}^{i}}(Q+\lambda)^{-1}\right\|_{B_{q}}+\left\|A(Q+\lambda)^{-1}\right\|_{B_{q}} \leq C
$$

Let $E$ be a Banach space and $X_{q}=X_{q}(G)=\left(L^{q}(G ; E)\right)^{n}$ denote the class of $E$-valued system of function $f=\left(f_{1}(x), f_{2}(x), \ldots, f_{n}(x)\right)$ with norm

$$
\|f\|_{X_{q}}=\left(\sum_{i=1}^{n}\left\|f_{i}\right\|_{L^{q(G ; E)}}^{q}\right)^{\frac{1}{q}}, \quad q \in(1, \infty)
$$


$X_{q \sigma}=L_{\sigma}^{q}(G ; E)$ denote the $E$-valued solenoidal space and $A$ be a positive operator in $E$. The spaces $\left(L^{s, q}(G ; E)\right)^{n},\left(L^{s, q}(G ; E(A), E)\right)^{n}$ will be denoted by $X_{q}^{s}(G)$ and $X_{q}^{s}(G, A)$.

Consider the problem

$$
\begin{aligned}
& \sum_{k=1}^{n} a_{k}(x) \frac{\partial^{2} u}{\partial x_{k}^{2}}+(A(x)+\lambda) u+\sum_{k=1}^{n} A_{k}(x) \frac{\partial u}{\partial x_{k}}=f(x), \quad x \in G, \\
& \sum_{i=0}^{m_{k j}}\left[\alpha_{k j i} \frac{\partial^{i} u}{\partial x_{k}^{i}}\left(G_{k 0}\right)+\beta_{k j i} \frac{\partial^{i} u}{\partial x_{k}^{i}}\left(G_{k b}\right)\right]=0, \quad k=1,2, \ldots, n, j=1,2,
\end{aligned}
$$

where $f=\left(f_{1}(x), f_{2}(x), \ldots, f_{n}(x)\right), A(x)$ and $A_{k}(x)$ are linear operators in a Banach space $E, a_{k}$ are complex-valued functions, and $\lambda$ is a complex parameter. From Theorem 3.1 we obtain the following result.

Result 3.2 Suppose Condition 3.1 is satisfied. Then problem (3.2) has a unique solution $u \in X_{q}^{2}(G, A)$ for $f \in X_{q}$ and for sufficiently large $\lambda \in S_{\varphi}$. Moreover, the following coercive uniform estimate holds:

$$
\sum_{k=1}^{n} \sum_{i=0}^{2}|\lambda|^{1-\frac{i}{2}}\left\|\frac{\partial^{i} u}{\partial x_{k}^{i}}\right\|_{X_{q}}+\|A u\|_{X_{q}} \leq C\|f\|_{X_{q}} .
$$

Consider the differential operator $B=B_{q}$ in $X_{q}$ generated by problem (3.2), for $\lambda=0$, i.e.,

$$
D(B)=X_{q}^{2}(G, A), \quad B u=\sum_{k=1}^{n} a_{k}(x) \frac{\partial^{2} u}{\partial x^{2}}+A(x)+\sum_{k=1}^{n} A_{k}(x) \frac{\partial u}{\partial x_{k}} .
$$

From Result 3.2 we obtain the following uniform coercive estimate:

$$
\sum_{k=1}^{n} \sum_{i=0}^{2}|\lambda|^{1-\frac{i}{2}}\left\|\frac{\partial^{i}}{\partial x_{k}^{i}}(B+\lambda)^{-1}\right\|_{B\left(X_{q}\right)}+\left\|A(B+\lambda)^{-1}\right\|_{B\left(X_{q}\right)} \leq C .
$$

Consider the space

$$
\begin{aligned}
& Y_{q}(A)=\left\{u \in X_{q}(E(A)), \operatorname{div} u \in L^{q}(G ; E)\right\}, \\
& \|u\|_{Y_{q}(A)}=\left(\|u\|_{X_{q}(E(A))}^{q}+\|\operatorname{div} u\|_{L^{q}(G ; E)}^{q}\right)^{\frac{1}{q}} .
\end{aligned}
$$

$Y_{q}$ becomes a Banach space with this norm.

It is known that (see, e.g., $[3,5])$ the vector field $u \in\left(L^{q}(G)\right)^{n}$ has a Helmholtz decomposition. In the following theorem we generalize this result for the $E$-valued function space $X_{q}$.

Theorem 3.2 Let $E$ be an UMD space and $q \in(1, \infty)$. Then $u \in X_{q}$ has a Helmholtz decomposition, i.e., there exists a linear bounded projection operator $P_{q}$ from $X_{q}$ onto $X_{q \sigma}$ with null space $N\left(P_{q}\right)=\left\{\nabla \varphi \in X_{q}: \varphi \in L_{l o c}^{q}(G ; E)\right\}$. In particular, all $u \in X_{q}$ have a unique decomposition $u=u_{0}+\nabla \varphi$ with $u_{0} \in X_{q \sigma}, u_{0}=P_{q} u$ so that

$$
\|\nabla \varphi\|_{X_{q}}+\left\|u_{0}\right\|_{X_{q}} \leq C\|u\|_{X_{q}} .
$$

Moreover, $\left(X_{q \sigma}\right)^{*}=X_{q^{\prime} \sigma}$, where $\frac{1}{q}+\frac{1}{q^{\prime}}=1$. 
For proving Theorem 3.2 we need some lemmas.

Consider the equation

$$
-\Delta u+A(x) u=f(x), \quad x \in G .
$$

Lemma 3.1 Let $E$ be an $U M D$ space, $A$ a R-positive operator in $E$ and $q \in(1, \infty)$. Then, for $f \in X_{q}^{-1}$, problem (3.2) has a unique solution $u \in X_{q}^{1}(A)$ and the following coercive estimate holds:

$$
\|u\|_{X_{q}^{1}(G)}+\|A u\|_{X_{q}(G)} \leq C\|f\|_{X_{q}^{-1}(G)}
$$

Proof Consider the problem

$$
-\Delta u+A u=\tilde{f}(x), \quad x \in R^{n}
$$

where $\tilde{f}(x)$ is an extension of the function $f(x)$ on $R^{n}$. Then, by using the Fourier inversion formula, operator-valued multiplier theorems in $L^{q}$ spaces, and by reasoning as in [17, Theorem 3.2] we see that problem (3.7) has a unique solution $\tilde{u} \in X_{q}^{1}\left(R^{n}, A\right)$ for $f \in X_{q}^{-1}\left(R^{n} ; E\right)$ and the following coercive estimate holds:

$$
\|\tilde{u}\|_{X_{q}^{1}\left(R^{n}\right)}+\|A \tilde{u}\|_{X_{q}\left(R^{n}\right)} \leq C\|\tilde{f}\|_{X_{q}^{-1}\left(R^{n}\right)} .
$$

This fact implies that the function $u$ which is a restriction of $\tilde{u}$ on $G$ is a solution of problem (3.5). The estimate (3.6) is obtained from the above estimate.

Let $v=\left(v_{1}, v_{2}, \ldots, v_{n}\right)$ be a unit normal to the boundary $\Gamma$ of the domain $G$ and $f_{v}$ is a normal component of $f=\left(f_{1}, f_{2}, \ldots, f_{n}\right) \in X_{q}(G)$ on $\Gamma$, i.e.,

$$
f_{v}=\left.\left(\sum_{k=1}^{n} v_{k} f_{k}\right)\right|_{\Gamma} .
$$

Here and hereafter $E^{*}$ will denoted the conjugate of $E$, and $(, \cdot)($ resp. $\langle, \cdot\rangle)$ denotes the duality pairing of functions on $G$ (resp. $\Gamma$ ).

By reasoning as in [3, Lemma 2] we get the following.

Lemma 3.2 $C_{0}^{\infty}(G ; E)$ is dense in $Y_{q}(A)$.

Proposition 3.1 There exists a unique bounded linear operator $u \rightarrow u_{\nu}$ from $Y_{q}(A), q \in$ $(1, \infty)$ onto

$$
W_{q}(\Gamma)=W^{-1 / q, q}\left(\Gamma,\left(E\left(A^{*}\right), E^{*}\right)_{q, 1 / q^{\prime}}, E^{*}\right)
$$

such that

$$
\left(u_{v},\left.v\right|_{\Gamma}\right)=(\operatorname{div} u, v)+(u, \nabla v), \quad v \in W^{1, q^{\prime}}(G, E(A), E)
$$


and the following estimate holds:

$$
\left\|u_{v}\right\|_{W_{q}(\Gamma)} \leq C\left(\|u\|_{X_{q}}+\|\operatorname{div} u\|_{L^{q}(G ; E)}\right)
$$

where

$$
\frac{1}{q}+\frac{1}{q^{\prime}}=1
$$

Proof For $u \in Y_{q}(A)$ consider the linear form

$$
T_{u}(v)=(\operatorname{div} u, \Phi)+(u, \nabla \Phi), \quad \Phi \in W^{q^{\prime}, 1}(G, E(A), E), \quad \Phi_{v}=v .
$$

By virtue of the trace theorem in $W^{1, q}(0, a ; E(A), E)$, the interpolation of intersection and dual spaces (see, e.g., $[13, \$ 1.8 .2,1.12 .1,1.11 .2]$ ) and by a localization argument we obtain the result that the operator $\Phi \rightarrow \Phi_{\nu}$ is a bounded linear and surjective from $W^{1, q^{\prime}}(G ; E(A), E)$ onto

$$
Z(\Gamma)=W^{1-1 / q^{\prime}, q^{\prime}}\left(\Gamma,(E(A), E)_{q^{\prime}, 1 / q^{\prime}}, E\right) .
$$

Hence, we can find for each $v \in Z(\Gamma)$ an element $\Phi \in W^{1, q^{\prime}}(G ; E(A), E)$ so that

$$
\Phi_{\nu}=v,\|\Phi\|_{W^{1, q^{\prime}(G ; E(A), E)}} \leq C\|v\|_{Z(\Gamma)}
$$

Therefore, from (3.9) we get

$$
\begin{aligned}
\left|T_{u}(v)\right| & \leq\left(\|u\|_{X_{q}}+\|\operatorname{div} u\|_{L^{q}(G ; E)}\right)\|\Phi\|_{W^{1, q^{\prime}}(G ; E(A), E)} \\
& \leq C\left(\|u\|_{X_{q}}+\|\operatorname{div} u\|_{L^{q}(G ; E)}\right)\|v\|_{Z(\Gamma)} .
\end{aligned}
$$

This implies the existence of an element

$$
u_{\nu} \in(Z(\Gamma))^{*}=W^{-1 / q, q}\left(\Gamma,\left(E\left(A^{*}\right), E^{*}\right)_{q, 1 / q^{\prime}} E^{*}\right)
$$

such that

$$
\left\langle u_{v}, v\right\rangle=T_{u}(v) \quad \text { for } v \in Z(\Gamma)
$$

and

$$
\left\|u_{v}\right\|_{(Z(\Gamma))^{*}} \leq C\left(\|u\|_{X_{q}}+\|\operatorname{div} u\|_{L^{q}(G ; E)}\right) .
$$

Thus, we have proved the existence of the operator $u \rightarrow u_{v}$. The uniqueness follows from Lemma 3.2.

Proposition 3.1 implies the following. 
Result 3.3 Assume the conditions of Proposition 3.1 are satisfied. Then

$$
X_{q \sigma} \subset X_{q v}=\left\{u \in X_{q}, \operatorname{div} u=0, u_{v}=0\right\}
$$

and $X_{q \nu}$ is a closed subspace of $X_{q}$.

$$
\text { Let } f \in X_{q} \text { and } f=\left(f_{1}(x), f_{2}(x), \ldots, f_{n}(x)\right) \text {. Consider the following problem: }
$$

$$
\begin{aligned}
-\Delta u+A u & =\operatorname{div} f(x), \quad x \in G, \\
\frac{\partial u}{\partial x_{k}}\left(G_{k 0}\right) & =f_{k}\left(G_{k 0}\right), \quad \frac{\partial u}{\partial x_{k}}\left(G_{k b}\right)=f_{k}\left(G_{k b}\right), \quad k=1,2, \ldots, n .
\end{aligned}
$$

Lemma 3.3 Let $E$ be an UMD space, $A$ a R-positive operator in $E$ and $q \in(1, \infty)$. Then, for $f \in X_{q}$, problem (3.10) has a unique solution $u \in W^{1, q}(G ; E(A), E)$ and the coercive estimate holds

$$
\|u\|_{W^{1, q(G ; E)}}+\|A u\|_{L^{q}(G ; E)} \leq C\|\operatorname{div} f\|_{W^{-1, q(G ; E)}}+\left\|f_{v}\right\|_{W_{q}(\Gamma)} .
$$

Proof Consider the equation

$$
-\Delta v+A v=\operatorname{div} f(x), \quad x \in G .
$$

By Lemma 3.1, problem (3.12) has a unique solution $v \in L^{1, q}(G ; E(A), E)$ for $f \in X_{q}$ and the following estimate holds:

$$
\|v\|_{L^{1, q}(G ; E)}+\|A v\|_{L^{q}(G ; E)} \leq C\|\operatorname{div} f\|_{L^{-1, q}(G ; E)} .
$$

Consider now the BVP

$$
\begin{aligned}
& -\Delta w+A w=0, \quad x \in G, \\
& \frac{\partial w}{\partial x_{k}}\left(G_{k 0}\right)=f_{k}\left(G_{k 0}\right)-\frac{\partial v}{\partial x_{k}}\left(G_{k 0}\right), \\
& \frac{\partial w}{\partial x_{k}}\left(G_{k b}\right)=f_{k}\left(G_{k b}\right)-\frac{\partial v}{\partial x_{k}}\left(G_{k b}\right), \quad k=1,2, \ldots, n .
\end{aligned}
$$

By using Theorem 3.1, Result 3.3, and Proposition 3.1 we conclude that problem (3.14) for $f \in X_{q}$ has a unique solution $w \in W^{1, q}(G ; E(A), E)$ and the following coercive estimate holds:

$$
\|w\|_{W^{1, q}(G ; E)}+\left\|A^{\frac{1}{2}} w\right\|_{L^{q}(G ; E)} \leq C\left\|f_{v}\right\|_{W_{q}(\Gamma)} .
$$

Then we conclude that problem (3.10) has a unique solution $\varphi(x)=v(x)+w(x)$ and (3.13), (3.15) imply the estimate (3.11).

Result 3.4 For the case of $A=\varkappa>0$ we obtain from (3.9), (3.11), and (3.12) that the problem

$$
(-\Delta+\varkappa) w=0, \quad x \in G
$$




$$
\begin{aligned}
& \frac{\partial w}{\partial x_{k}}\left(G_{k 0}\right)=f_{k}\left(G_{k 0}\right)-\frac{\partial v}{\partial x_{k}}\left(G_{k 0}\right), \\
& \frac{\partial w}{\partial x_{k}}\left(G_{k b}\right)=f_{k}\left(G_{k b}\right)-\frac{\partial v}{\partial x_{k}}\left(G_{k b}\right), \quad k=1,2, \ldots, n
\end{aligned}
$$

has a unique solution $w \in W^{1, q}(G ; E)$ for $f \in X_{q}$ and the following estimate holds:

$$
\|w\|_{W^{1, q}(G ; E)} \leq C\left\|f_{v}-\frac{\partial v}{\partial v}\right\|_{W^{-1 / q, q\left(\Gamma, E^{*}\right)}} .
$$

Result 3.5 For the case of $A=\varkappa>0$ we obtain from Lemma 3.1 and from (3.9), (3.10) that the problem

$$
(-\Delta+\varkappa) v=\operatorname{div} f(x), \quad x \in G
$$

has a unique solution $v \in W^{1, q}(G ; E)$ for $f \in X_{q}$ and the following estimate holds:

$$
\|v\|_{W^{1, q}(G ; E)} \leq C\|\operatorname{div} f\|_{X_{q}^{-1}}
$$

By (3.9), $\operatorname{div}(f-\nabla v)=0$. So, by (3.14) and Proposition 3.1 we get

$$
\|w\|_{W^{1, q(G ; E)}} \leq C\|f\|_{X_{q}}
$$

From Results 3.4, 3.5, and estimate (3.17) we obtain

Result 3.6 The problem

$$
\begin{aligned}
& (-\Delta+\varkappa) w=\operatorname{div} f(x), \quad x \in G, \\
& \frac{\partial w}{\partial x_{k}}\left(G_{k 0}\right)=f_{k}\left(G_{k 0}\right)-\frac{\partial v}{\partial x_{k}}\left(G_{k 0}\right), \\
& \frac{\partial w}{\partial x_{k}}\left(G_{k b}\right)=f_{k}\left(G_{k b}\right)-\frac{\partial v}{\partial x_{k}}\left(G_{k b}\right), \quad k=1,2, \ldots, n
\end{aligned}
$$

has a unique solution $u=w+v \in W^{1, q}(G ; E)$ for $f \in X_{q}$ and the estimate holds

$$
\|u\|_{W^{1, q(G ; E)}} \leq C\|f\|_{X_{q}} .
$$

Consider the operator $P=P_{q}$ defined by

$$
D(P)=X_{q}, \quad P f=f-\operatorname{grad}(v+w),
$$

where $w$ and $v$ are solutions of problems (3.18), (3.13), respectively. It is clear that we have the following.

Lemma 3.4 Let $E$ be an $U M D$ space and $q \in(1, \infty)$. Then $P_{q} X_{q}$ is a closed subspace of $X_{q}$.

Lemma 3.5 Let E be an UMD space and $q \in(1, \infty)$. Then the operator $P_{q}$ is a bounded linear operator in $X_{q}$ and $P f=f$ if $\operatorname{div} f(x)=0$. 
Proof The linearity of the operator $P$ is clear by construction. Moreover, by Result 3.6 we have

$$
\|P f\|_{X_{q}} \leq\|f\|_{X_{q}}+\|\operatorname{grad} u\|_{X_{q}} \leq C\|f\|_{X_{q}} .
$$

If $\operatorname{div} f(x)=0$ then by Result 3.3 we get $v=0$. Moreover, by the estimate (3.20) we obtain $w=0$, i.e., $P f=f$.

Lemma 3.6 Assume $E$ is an $U M D$ space and $q \in(1, \infty)$. Then the conjugate of $P_{q}$ is defined as $P_{q}^{*}=P_{q^{\prime}}, \frac{1}{q}+\frac{1}{q^{\prime}}=1$ and is bounded linear in $\left(L^{q^{\prime}}\left(G ; E^{*}\right)\right)^{n}$.

Proof It is known (see, e.g., $[13,18])$ that the dual space of $L^{q}(G ; E)$ is $L^{q^{\prime}}\left(G ; E^{*}\right)$. Since $C_{0}^{\infty}\left(G ; E^{*}\right)$ is dense in $L^{q^{\prime}}\left(G ; E^{*}\right)$ we have only to show $P_{q}^{*} \varphi=P_{q^{\prime}} \varphi$ for any $\varphi \in\left(C_{0}^{\infty}\left(G ; E^{*}\right)\right)^{n}$. But this is deriving by reasoning as in [3, Lemma 5]. Moreover, by Lemma 3.5 the dual operator $P_{q}^{*}$ is a bounded linear in $L^{q^{\prime}}\left(G ; E^{*}\right)$.

Let

$$
\begin{aligned}
& W_{q}=\left\{\nabla \varphi: \varphi \in W^{q, 1}(G ; E)\right\}, \\
& \left(P_{q} X_{q}\right)^{\perp}=\left\{f \in\left(L^{q^{\prime}}\left(G ; E^{*}\right)\right)^{n},\langle f, v\rangle=0 \text { for any } v \in P_{q} X_{q}\right\} .
\end{aligned}
$$

From Lemmas 3.5, 3.6 we obtain the following.

Result 3.7 Assume $E$ is an UMD space and $q \in(1, \infty)$. Then any element $f \in X_{q}$ uniquely can be expressed as a sum of elements of $P_{q} X_{q}$ and $W_{q}$.

In a similar way as Lemmas 6, 7 of [3] we obtain, respectively, the following.

Lemma 3.7 Assume $E$ is an UMD space and $q \in(1, \infty)$. Then

$$
\left(P_{q} X_{q}\right)^{\perp}=W_{q^{\prime}}, \quad \frac{1}{q}+\frac{1}{q^{\prime}}=1 .
$$

Lemma 3.8 Assume E is an UMD space and $q \in(1, \infty)$. Then

$$
X_{q \sigma}^{\perp}=W_{q^{\prime}}, \quad \frac{1}{q}+\frac{1}{q^{\prime}}=1 .
$$

Now we are ready to prove Theorem 3.2.

Proof of Theorem 3.2 From Lemmas 3.7, 3.8 we get $X_{q \sigma}=\left(P_{q} X_{q}\right)^{\perp}$. Then, by construction of $P_{q}$, we have $X_{q}=X_{q \sigma} \oplus W_{q}$. By Lemmas 3.3, 3.5, we obtain the estimate (3.4). Moreover, by Lemma 3.4, $W_{q}$ is a close subspace of $X_{q}$. Then it is known that the dual space of the quotient space $X_{q} / W_{q}$ is $W_{q}^{\perp}$. In view of first assertion we have $X_{q} / W_{q}=X_{q \sigma}$ and by Lemma 3.8 we obtain the second assertion. 
Theorem 3.3 Let Condition 3.1 hold. Then problem (1.7)-(1.8) has a unique solution $u \in$ $X_{q}^{2}$ for $f \in X_{q}, \varphi \in W^{1, q}(G ; E), \lambda \in S_{\psi, \varkappa}$, and the following coercive uniform estimate holds:

$$
\sum_{k=1}^{n} \sum_{i=0}^{2} \lambda^{1-\frac{i}{2}}\left\|\frac{\partial^{i} u}{\partial x_{k}^{i}}\right\|_{X_{q}}+\|A u\|_{X_{q}}+\|\nabla \varphi\|_{X_{q}} \leq C\|f\|_{X_{q}}
$$

Proof By virtue of Result 3.2, we find that problem (3.2) has a unique solution $u \in X_{q}^{2}(G, A)$ for $f \in X_{q}$ and for sufficiently large $\lambda \in S_{\varphi}$. Moreover, the following coercive uniform estimate holds:

$$
\sum_{k=1}^{n} \sum_{i=0}^{2}|\lambda|^{1-\frac{i}{2}}\left\|\frac{\partial^{i} u}{\partial x_{k}^{i}}\right\|_{X_{q}}+\|A u\|_{X_{q}} \leq C\|f\|_{X_{q}} .
$$

By applying the operator $P_{q}$ to problem (1.7)-(1.8) we get the Stokes problem (1.9)-(1.10). It is clear that

$$
D\left(O_{q}\right)=D(B) \cap X_{q \sigma},
$$

where $O_{q}$ is the Stokes operator and $B$ is a operator generated by problem (3.2) for $\lambda=0$. Then by Theorem 3.2 we obtain the assertion.

Result 3.8 From Result 3.2 we find that $O=O_{q}$ is a positive operator in $X_{q}$ and $-O$ generate a bounded holomorphic semigroup $S(t)=\exp (-O t)$ for $t>0$.

In a similar way as in [6] we show the following.

Proposition 3.2 The following estimate holds:

$$
\left\|O^{\alpha} S(t)\right\| \leq C t^{-\alpha}
$$

for $\alpha \geq 0$ and $t>0$.

Proof From the estimate (3.3) we see that the operator $O$ is positive in $X_{q}$, i.e., for $\lambda \in S_{\psi, \varkappa}$, $0<\psi<\pi$ the following estimate holds:

$$
\left\|(O+\lambda)^{-1}\right\| \leq M|\lambda|^{-1}
$$

where the constant $M$ is independent of $\lambda$. Then, by using the Danford integral and operator calculus (see, e.g., in [12]), we obtain the assertion.

\section{Well-posedness of instationary Stokes problems with variable coefficients}

Let

$$
B(p, q)=L^{p}\left(0, T ; X_{q}\right), \quad D(p, q)=\left(X_{q}^{2}(A), X_{q}\right)_{\frac{1}{p}, p} .
$$

In this section, we will show the well-posedness of problem (1.1)-(1.2). 
Theorem 4.1 Then, for $f \in B(p, q), \varphi \in L_{p}\left(0, T ; W^{1, q}(G ; E)\right)$, and $a \in D(p, q), p, q \in(1, \infty)$, there is a unique solution $(u, \nabla \varphi)$ of problem (1.1)-(1.2) and the following estimate holds:

$$
\left\|\frac{\partial u}{\partial t}\right\|_{B(p, q)}+\sum_{k=1}^{n}\left\|\frac{\partial^{2} u}{\partial x_{k}^{2}}\right\|_{B(p, q)}+\|A u\|_{B(p, q)}+\|\nabla \varphi\|_{B(p, q)} \leq C\left(\|f\|_{B(p, q)}+\|a\|_{D(p, q)}\right) .
$$

Proof Problem (1.1)-(1.2) can be expressed as the following abstract parabolic problem:

$$
\frac{d u}{d t}+O u=f(t), \quad u(0)=a .
$$

If we put $E=X_{q}$ then by Proposition 3.2, operator $O$ is positive and generates a bounded holomorphic semigroup in $X_{q}$. Moreover, by using [17, Theorem 3.1] we see that the operator $O$ is $R$-positive in $E$. Since $E$ is a UMD space, in a similar way as in [13, Theorem 4.2] we see that for all $f \in L^{p}(0, T ; E)$ and $a \in(D(O), E)_{\frac{1}{p}, p}$ there is a unique solution $u \in W^{1, p}(0, T, D(O), E)$ of problem (4.2) so that the following estimate holds:

$$
\left\|\frac{d u}{d t}\right\|_{L^{p}(0, T ; E)}+\|O u\|_{L^{p}(0, T ; E)} \leq C\left(\|f\|_{L^{p}(0, T ; E)}+\|a\|_{(D(O), E)_{\frac{1}{p}, p}}\right) .
$$

From the estimates (3.3) and (4.3) we obtain the assertion.

Remark 4.1 There are a lot of positive operators in concrete Banach spaces. Therefore, putting in (1.7)-(1.8) and (1.1)-(1.3) concrete Banach spaces instead of $E$ and concrete positive differential, pseudo differential operators, or finite, infinite matrices, etc. instead of $A$, by virtue of Theorem 3.3 and Theorem 4.1 we can obtain the maximal regularity properties of different class of stationary and instationary Stokes problems, respectively, which occur in numerous physics and engineering problems.

Let us now show some application of Theorem 3.3 and Theorem 4.1.

\section{Application}

Consider the stationary Stokes problem

$$
\begin{aligned}
& \sum_{k=1}^{n} a_{k}(x) \frac{\partial^{2} u(x, y)}{\partial x_{k}^{2}}+\sum_{|\beta| \leq 2 m} a_{\beta}(x, y) D_{y}^{\beta} u(x, y)+\nabla \varphi=f(x, y), \quad \operatorname{div} u=0, \\
& \sum_{i=0}^{m_{k j}}\left[\alpha_{k j i} \frac{\partial^{i} u}{\partial x_{k}^{i}}\left(G_{k 0}, y\right)+\beta_{k j i} \frac{\partial^{i} u}{\partial x_{k}^{i}}\left(G_{k b}, y\right)\right]=0, \quad y \in \Omega, j=1,2, \\
& B_{j} u=\left.\sum_{|\beta| \leq m_{j}} b_{j \beta}(y) D_{y}^{\beta} u(x, y)\right|_{y \in \partial \Omega}=0, \quad x \in G, j=1,2, \ldots, m,
\end{aligned}
$$

where $f=\left(f_{1}(x, y, t), f_{2}(x, y, t), \ldots, f_{n}(x, y, t)\right)$ represents a given and

$$
u=\left(u_{1}(x, y), u_{2}(x, y), \ldots, u_{n}(x, y)\right), \quad \varphi=\varphi(x, y)
$$


are unknown functions;

$$
\begin{aligned}
& x=\left(x_{1}, x_{2}, \ldots, x_{n}\right) \in G=\prod_{k=1}^{n}\left(0, b_{k}\right), \quad y=\left(y_{1}, \ldots, y_{\mu}\right) \in \Omega \subset R^{\mu}, \\
& G_{k 0}=\left(x_{1}, x_{2}, \ldots, x_{k-1}, 0, x_{k+1}, \ldots, x_{n}\right), \quad p \in(1, \infty), \\
& G_{k b}=\left(x_{1}, x_{2}, \ldots, x_{k-1}, b_{k}, x_{k+1}, \ldots, x_{n}\right), \quad q \in(1, \infty), m_{k j} \in\{0,1\},
\end{aligned}
$$

$\alpha_{k j i}, \beta_{k j i}$ are complex numbers, $a_{\alpha}, b_{j \beta}$ are complex-valued functions, $D_{j}=-i \frac{\partial}{\partial y_{j}}$.

Let $\tilde{\Omega}=G \times \Omega, \mathbf{p}=\left(p_{1}, p\right)$. Now $L^{\mathbf{P}}(\tilde{\Omega})$ will denote the space of all $\mathbf{p}$-summable scalarvalued functions with mixed norm i.e., the space of all measurable functions $f$ defined on $\tilde{\Omega}$, for which

$$
\|f\|_{L \mathbf{P}(\tilde{\Omega})}=\left(\int_{G}\left(\int_{\Omega}|f(x, y)|^{p_{1}} d x\right)^{\frac{p}{p_{1}}} d y\right)^{\frac{1}{p}}<\infty .
$$

Analogously, $W^{m, \mathbf{p}}(\tilde{\Omega})$ denotes the Sobolev space with corresponding mixed norm.

$$
X_{\mathbf{p}}=\left(L^{\mathbf{p}}(\tilde{\Omega})\right)^{n} \text { denotes the class of vector function }
$$

$$
f=\left(f_{1}(x), f_{2}(x), \ldots, f_{n}(x)\right)
$$

with norm

$$
\|f\|_{X_{\mathbf{p}}}=\sum_{i=1}^{n}\left\|f_{i}\right\|_{L(\mathbf{p}(G ; E)}
$$

and $X_{\mathbf{p}}^{2,2 m}=\left(W^{2,2 m, \mathbf{p}}(\tilde{\Omega})\right)^{n}$, where $W^{2,2 m, \mathbf{p}}(\tilde{\Omega})$ is the anisotropic Sobolev space with mixed norm.

From Theorem 3.3 we obtain the following.

\section{Theorem 5.1 Let the following conditions be satisfied:}

(1) $\Omega$ is a domain in $R^{\mu}$ with sufficiently smooth boundary $\partial \Omega, a_{\alpha}(x, y) \in C(\bar{\Omega})$, $a_{\alpha}\left(G_{i 0}, y\right)=a_{\alpha}\left(G_{i b}, y\right)$ for each $|\alpha|=2 m, y \in \Omega, a_{\alpha} \in\left[L_{\infty}+L_{r_{k}}\right](\Omega)$ for each $|\alpha|=k<2 m$ with $r_{k} \geq p_{1}, p_{1} \in(1, \infty)$ and $2 m-k>\frac{l}{r_{k}}, v_{\alpha} \in L_{\infty} ;$

(2) $b_{j \beta} \in C^{2 m-m_{j}}(\partial \Omega)$ for each $j, \beta, m_{j}<2 m, p \in(1, \infty)$;

(3) for $y \in \bar{\Omega}, \xi \in R^{\mu}, \eta \in S\left(\varphi_{1}\right), \varphi_{1} \in\left[0, \frac{\pi}{2}\right),|\xi|+|\eta| \neq 0$ let

$$
\eta+\sum_{|\alpha|=2 m} a_{\alpha}(y) \xi^{\alpha} \neq 0
$$

(4) for each $x_{0} \in \partial \Omega$ the local BVPs in local coordinates corresponding to $x_{0}$

$$
\begin{aligned}
& \eta+\sum_{|\alpha|=2 m} a_{\alpha}\left(x_{0}, \xi^{\prime}, D_{\mu}\right) \vartheta(y)=0, \quad y>0, \\
& B_{j 0} \vartheta=\left.\sum_{|\beta|=m_{j}} b_{j \beta}\left(x_{0}, \xi^{\prime}, D_{\mu}\right) \vartheta(y)\right|_{y=0}=h_{j}, \quad j=1,2, \ldots, m
\end{aligned}
$$


has a unique solution $\vartheta \in C_{0}\left(R_{+}\right)$for all $h=\left(h_{1}, h_{2}, \ldots, h_{m}\right) \in R^{m}$ and for $\xi^{\prime} \in R^{\mu-1}$ with $\left|\xi^{\prime}\right|+|\eta| \neq 0$;

(5) $a_{k} \in C(\bar{G}), a_{k}\left(G_{i 0}, y\right)=a_{k}\left(G_{i b}, y\right), a_{k} \neq 0, a_{k} \in S\left(\varphi_{0}\right) \cap \mathbb{C} / \mathbb{R}_{-}$for all $x \in G, \varphi+\varphi_{0}<\pi$, $y \in \Omega, k, i=1,2, \ldots, n$.

Then, problem (5.1)-(5.3) has a unique solution $u \in X_{\mathbf{p}}^{2,2 m}$ for $f \in X_{\mathbf{p}}, \varphi \in W^{1, \mathbf{p}}(\tilde{\Omega} ; E)$, $\lambda \in S_{\psi, \varkappa}$, and the following coercive uniform estimate holds:

$$
\sum_{k=1}^{n} \sum_{i=0}^{2}|\lambda|^{1-\frac{i}{2}}\left\|\frac{\partial^{i} u}{\partial x_{k}^{i}}\right\|_{X_{\mathbf{p}}}+\sum_{|\alpha|=2 m}\left\|D_{y}^{\alpha} u\right\|_{X_{\mathbf{p}}}+\|\nabla \varphi\|_{X_{\mathbf{p}}} \leq C\|f\|_{X_{\mathbf{p}}} .
$$

Proof Let $E=L^{p_{1}}(\Omega)$. By virtue of [18, Theorem 4.5.2] $L^{p_{1}}(\Omega)$ is an UMD space. Consider the operator $A$ which is defined by

$$
D(A)=W^{2 m, p_{1}}\left(\Omega ; B_{j} u=0\right), \quad A u=\sum_{|\beta| \leq 2 m} a_{\beta}(y) D^{\beta} u(y) .
$$

Problem (5.1)-(5.3) can be rewritten in the form of (1.7)-(1.8) for $A_{k}=0, k=1,2, \ldots, n$, where $u(x)=u(x, \cdot)$ and $f(x)=f(x, \cdot)$ are functions with values in $E=L^{p_{1}}(\Omega)$. In view of [14, Theorem 8.2] the problem

$$
\begin{aligned}
& \eta u(y)+\sum_{|\beta| \leq 2 m} a_{\beta}(x, y) D^{\beta} u(y)=f(y), \\
& B_{j} u=\sum_{|\beta| \leq m_{j}} b_{j \beta}(y) D^{\beta} u(y)=0, \quad j=1,2, \ldots, m
\end{aligned}
$$

has a unique solution for $f \in L^{p_{1}}(\Omega)$ and $\arg \eta \in S\left(\varphi_{1}\right),|\eta| \rightarrow \infty$, and the operator $A$ is $R$-positive in $L^{p_{1}}$. Hence, all conditions of Theorem 3.2 are satisfied, i.e., we obtain the assertion.

Consider now the instationary Stokes problem

$$
\begin{aligned}
& \frac{\partial u}{\partial t}+\sum_{k=1}^{n} a_{k}(x) \frac{\partial^{2} u}{\partial x_{k}^{2}}+\sum_{|\beta| \leq 2 m} a_{\beta}(x, y) D_{y}^{\beta} u+\nabla \varphi=f(x, y, t), \quad \operatorname{div} u=0, \\
& \sum_{i=0}^{m_{k j}}\left[\alpha_{k j i} \frac{\partial^{i} u}{\partial x_{k}^{i}}\left(G_{k 0}, y, t\right)+\beta_{k j i} \frac{\partial^{i} u}{\partial x_{k}^{i}}\left(G_{k b}, y, t\right)\right]=0, \quad j=1,2, \\
& u(x, y, 0)=a(x, y), \quad t \in(0, T), x \in G, y \in \Omega, \\
& B_{j} u=\left.\sum_{|\beta| \leq m_{j}} b_{j \beta}(y) D_{y}^{\beta} u(x, y, t)\right|_{y \in \partial \Omega}=0, \quad x \in G, j=1,2, \ldots, m,
\end{aligned}
$$

where $f(x, y, t), u=(x, y, t)$ are data and solution vector-functions, respectively.

From Theorem 4.1 and Theorem 5.1 we obtain the following.

Theorem 5.2 Assume all conditions of Theorem 5.1 are satisfied $p, p_{1}, q \in(1, \infty)$. Then, for $f \in L^{q}\left(0, T ; X_{\mathbf{p}}\right)=B\left(p, p_{1}, q\right), \varphi \in L^{q}\left(0, T ; W^{1, \mathbf{p}}(\tilde{\Omega})\right)$, and $a \in\left(X_{\mathbf{p}}^{2,2 m}, X_{\mathbf{p}}\right)_{\frac{1}{q}, q}=D\left(p, p_{1}, q\right)$, 
there is a unique solution $(u, \nabla \varphi)$ of problem (5.6)-(5.9) and the following estimate holds:

$$
\begin{gathered}
\left\|\frac{\partial u}{\partial t}\right\|_{B\left(p, p_{1}, q\right)}+\sum_{k=1}^{n}\left\|\frac{\partial^{2} u}{\partial x_{k}^{2}}\right\|_{B\left(p, p_{1}, q\right)}+\sum_{|\alpha|=2 m}\left\|D_{y}^{\alpha} u\right\|_{B\left(p, p_{1}, q\right)} \\
+\|\nabla \varphi\|_{B\left(p, p_{1}, q\right)} \leq C\left(\|f\|_{B\left(p, p_{1}, q\right)}+\|a\|_{D\left(p, p_{1}, q\right)}\right) .
\end{gathered}
$$

\section{Competing interests}

The author declares that they have no competing interests.

\section{Received: 30 September 2013 Accepted: 21 April 2014 Published: 02 May 2014}

\section{References}

1. Amann, H: On the strong solvability of the Navier-Stokes equations. J. Math. Fluid Mech. 2, $16-98$ (2000)

2. Giga, $Y$, Sohr, H: Abstract $L_{p}$ estimates for the Cauchy problem with applications to the Navier-Stokes equations in exterior domains. J. Funct. Anal. 102, $72-94$ (1991)

3. Fujiwara, D, Morimoto, H: An $L_{r}$-theorem of the Helmholtz decomposition of vector fields. J. Fac. Sci., Univ. Tokyo, Sect. 1A, Math. 24, 685-700 (1977)

4. Fujita, H, Kato, T: On the Navier-Stokes initial value problem I. Arch. Ration. Mech. Anal. 16, 269-315 (1964)

5. Farwing, R, Sohr, H: Generalized resolvent estimates for the Stokes system in bounded and unbounded domains. J. Math. Soc. Jpn. 46(4), 607-643 (1994)

6. Kato, T: Strong $L_{p}$-solutions of the Navier-Stokes equation in $R^{m}$, with applications to weak solutions. Math. Z. 187 471-480 (1984)

7. Ladyzhenskaya, OA: The Mathematical Theory of Viscous Incompressible Flow. Gordon \& Breach, New York (1969)

8. Solonnikov, V: Estimates for solutions of nonstationary Navier-Stokes equations. J. Sov. Math. 8, 467-529 (1977)

9. Sobolevskii, PE: Study of Navier-Stokes equations by the methods of the theory of parabolic equations in Banach spaces. Sov. Math. Dokl. 5, 720-723 (1964)

10. Teman, R: Navier-Stokes Equations. North-Holland, Amsterdam (1984)

11. Shakhmurov, V: Coercive boundary value problems for regular degenerate differential-operator equations. J. Math. Anal. Appl. 292(2), 605-620 (2004)

12. Denk, R, Hieber, M, Pruss, J: R-boundedness, Fourier multipliers and problems of elliptic and parabolic type. Mem. Am. Math. Soc. 166, 788 (2005)

13. Triebel, H: Interpolation Theory. Function Spaces. Differential Operators. North-Holland, Amsterdam (1978)

14. Ashyralyev, A: On well-posedeness of the nonlocal boundary value problem for elliptic equations. Numer. Funct. Anal. Optim. 24(1-2), 1-15 (2003)

15. Triebel, H: Fractals and Spectra: Related to Fourier Analysis and Function Spaces. Birkhäuser, Basel (1997)

16. Shakhmurov, VB: Embedding theorems and maximal regular differential operator equations in Banach-valued function spaces. J. Inequal. Appl. 4, 605-620 (2005)

17. Shakhmurov, VB: Linear and nonlinear abstract equations with parameters. Nonlinear Anal., Real World Appl. 73, 2383-2397 (2010)

18. Amann, H: Linear and Quasi-Linear Equations, vol. 1. Birkhäuser, Basel (1995)

19. Shakhmurov, VB, Shahmurova, A: Nonlinear abstract boundary value problems atmospheric dispersion of pollutants. Nonlinear Anal., Real World Appl. 11(2), 932-951 (2010)

20. Shakhmurov, VB: Separable anisotropic differential operators and applications. J. Math. Anal. Appl. 327(2), 1182-1201 (2006)

21. Shakhmurov, V: Parameter dependent Stokes problems in vector-valued spaces and applications. Bound. Value Probl. 2013, 172 (2013)

22. Weis, L: Operator-valued Fourier multiplier theorems and maximal $L_{p}$ regularity. Math. Ann. 319, 735-758 (2001)

23. Yakubov, S, Yakubov, Y: Differential-Operator Equations. Ordinary and Partial Differential Equations. Chapman \& Hall/CRC, Boca Raton (2000)

24. Lunardi, A: Analytic Semigroups and Optimal Regularity in Parabolic Problems. Birkhäuser, Basel (2003)

25. Skubachevskii, AL: Nonlocal boundary value problems. J. Math. Sci. 155(2), 199-334 (2008)

10.1186/1687-2770-2014-86

Cite this article as: Shakhmurov: Stokes operators with variable coefficients and applications. Boundary Value Problems 2014, 2014:86 